\title{
Intranasal fentanyl for pain control: current status with a focus on patient considerations
}

This article was published in the following Dove Press journal:

Patient Preference and Adherence

17 March 201 I

Number of times this article has been viewed

\section{Eric Prommer \\ Lisa Thompson}

Division of Hematology/Oncology, Mayo Clinic College of Medicine, Mayo Clinic Hospital, Scottsdale, AZ, USA
Correspondence: Eric Prommer Director of Palliative Care, Division of Hematology/Oncology, Assistant Professor, Mayo Clinic College of Medicine, Mayo Clinic Hospital, 5777 E. Mayo Blvd, Room 4E I07, Scottsdale, AZ 85054, USA

Tel +l 4803422000

Fax +I 4803422088

Email prommer.eric@mayo.edu
Abstract: Of several newer delivery systems under development and investigation for the administration of opioids, the intranasal route has received a substantial amount of attention. Intranasal administration is a convenient form of delivery that is applicable to several opioids. It has the potential for self-administration, combined with a rapid onset of action, allowing for patient-controlled analgesia. In clinical practice, intranasal administration has been found to be a reliable drug delivery method that is familiar to patients. Intranasal opioids have proven to be useful in both in-hospital and out-of-hospital pain management settings. Fentanyl, a highly lipophilic step 3 opioid, has been evaluated for intranasal administration. The purpose of this review is to examine the role of the nasal route of opioid administration and examine the evidence base for the use of fentanyl intranasally.

Keywords: fentanyl, intranasal, pain, breakthrough pain

\section{Introduction}

Pain is a common symptom in the general population and has a substantial impact on quality of life. Pain can be a major burden to patients and society. Opioids are among the most potent analgesics available and are the cornerstone of treatment for moderate to severe pain associated with cancer. ${ }^{1}$ Opioids can decrease pain and improve function. ${ }^{2}$ Most commonly recommended is the oral route of administration. There are times when nonoral routes of administration are necessary, such as in patients with advanced illness or in the postoperative period. Nonoral routes of administration have been shown to be more effective than the oral route by providing enhanced absorption and avoidance of first-pass effects. The nonoral routes of opioid administration include parenteral (subcutaneous, intravenous), rectal, sublingual, and transmucosal. The parenteral routes of administration are associated with rapid onset of action but can be associated with pain and inconvenience. The rectal route of administration has been evaluated for opioid administration and, although effective, can have limitations in terms of dignity for the patient and lack of uniform absorption. The sublingual and transmucosal routes are effective nonoral routes that have the advantage of avoiding presystemic elimination, yet can have suboptimal absorption due to swallowing. Lack of salivary production may impede the absorption of opioids when given by the transmucosal route.

The intranasal route has increasingly been viewed as a new alternative route for drug administration. The intranasal route is a convenient form of delivery that is applicable to several opioids and has the potential for self-administration, combined with a rapid onset of action. In clinical practice, intranasal administration has been 
found to be a reliable drug delivery method that is familiar to patients. Intranasal opioids have proven to be useful in both in-hospital and out-of-hospital pain management settings. Fentanyl (Figure 1) is a step 3 opioid that is used for acute and chronic pain of both malignant and nonmalignant origin. It is a lipophilic, short-acting, synthetic opioid with a piperidine chemical structure. ${ }^{3}$ Fentanyl can be administered by the transdermal, transmucosal, or sublingual routes, in addition to the traditional parenteral routes. Recently, fentanyl has been evaluated for intranasal administration. The purpose of this review is to examine the role of the nasal route of opioid administration and examine the evidence base for the use of fentanyl intranasally.

\section{Anatomy and physiology of the nose}

In adults, the nasal mucosa provides a large mucosal surface area $\left(150-180 \mathrm{~cm}^{2}\right)$ that is closely associated with high blood flow. ${ }^{4}$ This blood flow is greater per cubic centimeter of tissue than that of muscle, the brain, or the liver. ${ }^{4}$ The nose has a direct connection to the central nervous system via the olfactory route. Theoretically, the direct delivery of drugs to the brain allows lower doses of drugs to be used, with less delivery to nontargeted organs, and perhaps less toxic side effects. ${ }^{5}$ The nasal mucosa has a $\mathrm{pH}$ of 5.5-6.5, which maintains optimal function of glycoproteins to which drugs attach. ${ }^{4}$ Drug absorption through the nasal mucosa is also dependent on lipophilicity, drug ionization, and mucociliary clearance. ${ }^{6}$ These features make the nasal route a potentially useful route for the administration of drugs such as lipophilic opioids. The route avoids first-pass metabolism. ${ }^{4}$ The intranasal route of drug administration has been used for a variety of drugs, such as vaccines, ${ }^{7}$ opioids, benzodiazepines, migraine therapies, ${ }^{8}$ and gene therapy. ${ }^{5}$ Metabolic enzymes are present in the nasal mucosa and thus are able to metabolize drugs administered there. ${ }^{5}$

\section{Formulations}

Typical formulations of intranasal fentanyl consist of using an intravenous solution either directly or via spray devices. ${ }^{9}$ The ideal volume recommended is $0.15 \mathrm{~mL}$ in one or both nostrils. ${ }^{6}$ This is important, as run-off from the nasal cavity has the potential to be swallowed. Penetration enhancers have been used to overcome volume limitations, and additives such as polymers and gels or polysaccharides can enhance contact time, improving absorption. ${ }^{10}$ One study has demonstrated improved fentanyl absorption with polysaccharides such as pectin, chitosan, and chitosan-poloxamer compared

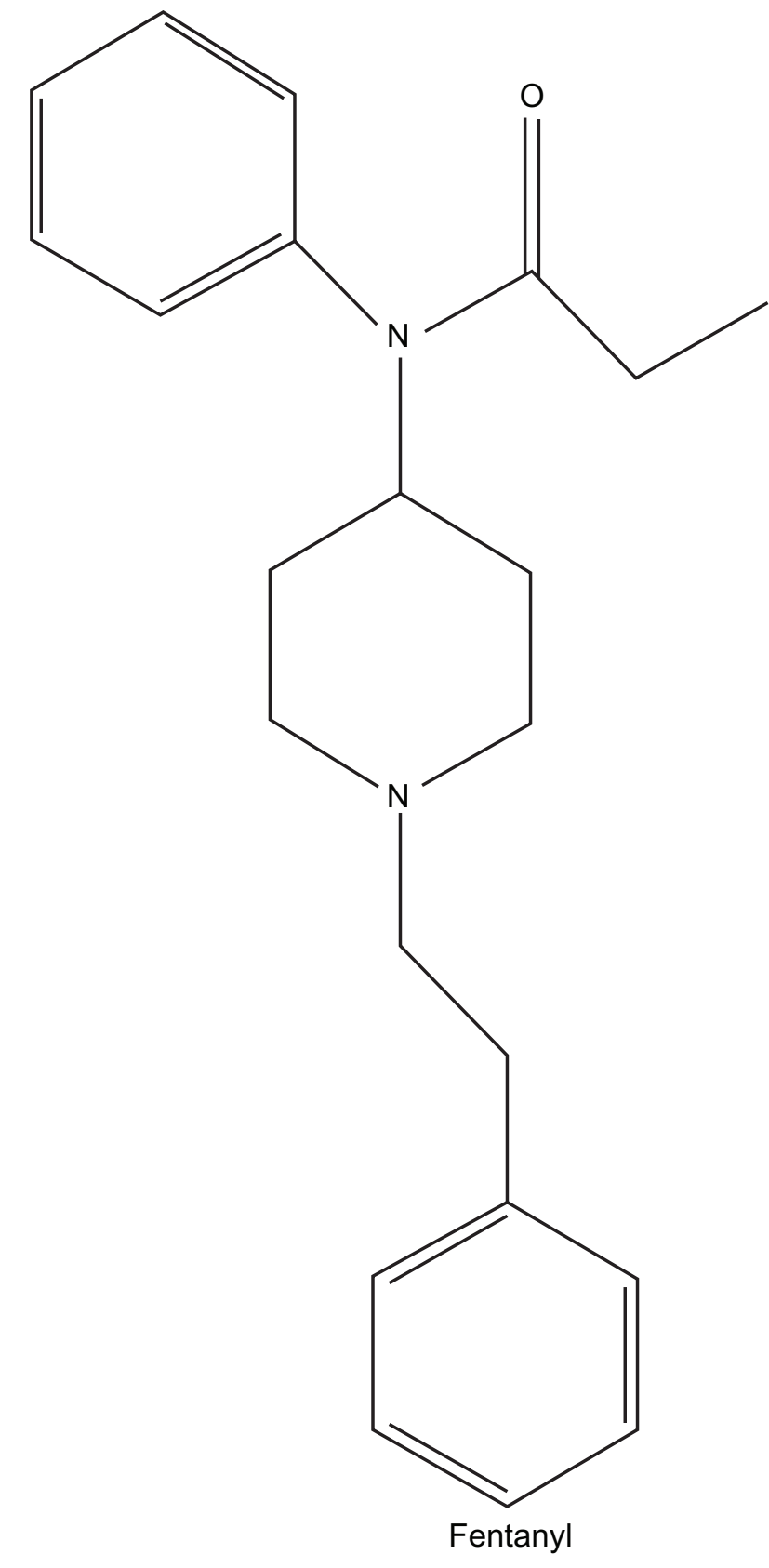

Figure I Fentanyl structure.

with transmucosal fentanyl. ${ }^{11}$ Biospheres may also increase residence time on the nasal mucosa. ${ }^{11}$

\section{Intranasal pharmacokinetics compared with other routes of fentanyl administration}

The pharmacology of several opioids has been evaluated after intranasal administration. ${ }^{6}$ Opioids that have been evaluated include the fentanyl series, oxycodone, methadone, buprenorphine, and diacetylmorphine. All the lipophilic 
opioids show rapid absorption, with time to maximum plasma concentration $\left(\mathrm{t}_{\max }\right)$ ranging from as short as 9 minutes for alfentanyl to up to 30 minutes for sufentanil. When administered via an intranasal spray, all of the opioids described reach $\mathrm{t}_{\max }$ in less than 30 minutes. Bioavailability of lipophilic opioids given intranasally is high and greater than the oral route for all step 3 opioids, with the exception of oxycodone. ${ }^{6}$ The pharmacokinetic data of all of the studies of opioids support the use of noninvasive intranasal drug delivery when a fast onset is indicated. When fentanyl is administered by the intranasal route, the bioavailability is nearly $70 \%$, with $\mathrm{t}_{\max }$ reached in 5-16 minutes. ${ }^{12}$ Fentanyl levels show dose dependency when administered intranasally. The absorption of fentanyl can be influenced by $\mathrm{pH}$, with greater absorption as the $\mathrm{pH}$ increases. ${ }^{13}$ Temperature can influence absorption, with greater permeation of fentanyl intranasally. ${ }^{14}$ The halflife is up to 65 minutes when given by intranasal route. Pharmacokinetic comparisons have been made between intranasal fentanyl and other routes of administration, such as transmucosally administered fentanyl. Transmucosal fentanyl has a decreased bioavailability and area under the curve compared with intranasal fentanyl. ${ }^{15}$ The half-life of transmucosal fentanyl is longer than intranasal fentanyl. ${ }^{15}$ Compared with intravenous fentanyl, the intranasal route has a slower onset of action. The half-life of the intravenous route is longer than that of the intranasal route. ${ }^{16}$

\section{Adverse effects}

When given intranasally, fentanyl can cause not only local effects specific to the intranasal route but also systemic adverse effects typical for opioids in general. ${ }^{6}$ The systemic effects of intranasally administered fentanyl can include dizziness, as well as sedation, nausea, and constipation. Local effects include nasal discomfort and irritation, the most common adverse effect with short-term use. There are fewer data on the long-term effects of intranasal opioid administration, but reports exist of epistaxis, pharyngitis, and sinus congestion. Respiratory depression does not occur more frequently with this route. ${ }^{6}$ Some indications are that polymer fentanyl combinations may prevent a higher incidence of adverse effects. ${ }^{17}$

\section{Drug interactions}

Fentanyl is metabolized by the cytochrome P450 3A4 liver isoenzyme system, and potent inhibitors of this enzyme may produce increased or prolonged opioid side effects. ${ }^{18}$ The concomitant use of other central nervous system depressants may produce additional sedative effects.

\section{Clinical trials}

Intranasal fentanyl has been evaluated for cancer-related breakthrough pain and postoperative pain and has been compared with morphine in several painful clinical conditions. There have been some studies in the management of symptoms in the hospice setting.

\section{Cancer-related breakthrough pain}

Breakthrough pain associated with cancer is defined as a transitory exacerbation of pain experienced by a patient who has relatively stable and adequately controlled baseline pain. ${ }^{19}$ The use of a lipophilic opioid such as fentanyl has been shown to be effective when given by other routes such as the transmucosal route ${ }^{20}$ and in formulations such as a buccal tablet, ${ }^{21}$ due to the lipophilic nature of the drug and its rapid onset of action. The rapid onset of action of fentanyl can match the rapid onset of action for most forms of breakthrough pain. The rapid onset of action by the intranasal route has been evaluated as another alternative route for the management of breakthrough pain.

\section{Intranasal fentanyl and placebo}

Intranasal fentanyl spray (INFS) at a dose range of 50-200 $\mu \mathrm{g}$ was evaluated for cancer-related breakthrough pain and compared with placebo. One multicenter, blinded, crossover trial ${ }^{22}$ focused on adult cancer patients who experienced at least three episodes of severe breakthrough pain per week, and up to a maximum of four episodes per day lasting longer than 15 minutes. Patients had already been receiving strong opioids such as oral morphine, oxycodone, or hydromorphone with dosages ranging from $60 \mathrm{mg}$ to $500 \mathrm{mg}$ per day, and transdermal fentanyl dosages ranging from $25 \mu \mathrm{g}$ to $200 \mu \mathrm{g}$ per hour. Patients were required to be at stable opioid dosing levels, with pain scores less than 4 on an 11-point numeric rating scale (NRS) for pain for entry into the trial. Because there is evidence that the breakthrough dose of fentanyl does not correlate with the amount of scheduled opioid, doses of intranasal fentanyl for breakthrough pain were individually titrated. A dose was considered successful if it could treat three of four episodes of breakthrough pain. Responses were measured by categorical scale $(0=$ poor; $1=$ fair; $2=$ good; 3 = very good; and $4=$ excellent). A successful dose had to get a score of 2 on the categorical scale 60 minutes after the breakthrough dose. There also had to be an absence of adverse effects to qualify for a successful breakthrough dose. Once the breakthrough dose was determined, 
randomization occurred to treatment sequences composed of two sets of four administrations (numbered 1-4 and 5-8), with each set comprising three INFS administrations and one placebo administration. Patients were instructed to treat eight episodes of breakthrough pain (to a maximum of four episodes daily) using the order in which the spray bottles were numbered (1-8). Patients recorded their pain in a diary using the 11-point NRS at times 10, 20, 40, and 60 minutes after administration. The main endpoint was the difference in the pain score at 10 minutes after administration (pain intensity difference [PID]10), with secondary endpoints being the sum of the pain intensities from baseline to 60 minutes after administration, and the general impression of efficacy at 60 minutes, using the aforementioned categorical scale. Clinically important PID10 scores are a decrease in pain of more than 2 points. Mean response rates were calculated for drug or placebo in each patient as well as reduction in pain intensities by more than $33 \%$ and more than $50 \%$, respectively. One-hundred and eleven patients were included in the study from an initial 120 patients. The results favored the intranasal fentanyl, as the PID10 was twice as great as for placebo $(P<0.001)$. The overall response rate for INFS was $51 \%$, with $20 \%$ of responses observed in the placebo arm. Adverse effects were observed in nearly $20 \%$ of patients, the most common being nausea $(4.5 \%)$ and vertigo $(1.8 \%)$.

\section{Fentanyl pectin formulation}

A multicenter, randomized, blinded, placebo-controlled study conducted by the Nasal Spray 043 Study Group ${ }^{23}$ evaluated the efficacy of fentanyl pectin nasal spray (FPNS) for cancer breakthrough pain. One-hundred and fourteen cancer patients who were taking at least $60 \mathrm{mg}$ of oral morphine or an equivalent opioid and who had one to four episodes of moderate to severe breakthrough pain were eligible for participation. Patients were titrated to the appropriate breakthrough dose (open label). Once a dose was identified, patients were randomized to receive 10 bottles, seven of which contained the effective dose and three contained placebo in a randomly assigned sequence. Patients could take up to four doses daily with 4 hours separated between doses. If the pain was not controlled in 30 minutes, or an episode of breakthrough pain occurred within the 4-hour period, patients could take their usual breakthrough medication. Pain relief was measured by NRS and categorical scale at baseline and at 5, 10, 15, 30, 45, and 60 minutes after administration. Patients had to rate overall satisfaction. Electronic diaries recorded information on the breakthrough doses. Adverse effect assessment included categorical scoring for local nasal effects.

Primary efficacy was measured by outcomes such as pain intensity, pain intensity differences from baseline, sum of pain intensity differences, pain relief, and total pain relief. Patients also evaluated ease and convenience by categorical scales (0-3). Seventy-two percent identified a breakthrough dose. Six percent withdrew due to lack of efficacy, and 5\% withdrew due to adverse effects. Baseline pain scores were comparable between placebo and study drug groups. Fentanyl was more effective in reducing pain intensity at all time points and had quicker onset of analgesic effects than placebo. One-third of FPNS-treated episodes had clinically meaningful reduction at 10 minutes ( $P=0.01$ vs placebo) and had an increase to $66 \%$ of episodes at 30 minutes $(P<0.0001$ vs placebo). There were more 2-point pain reductions with FPNS at each time point after dosing $(P<0.01)$. In addition, the FPNS group has a greater number of 1- or 2-point reductions at each time point. There was less need for additional breakthrough medications with FPNS $(P<0.001)$. Patient acceptability was better for the FPNS group at 30 and 60 minutes than for the placebo group. FPNS was also superior in terms of time to relief than placebo at 30 and 60 minutes $(P<0.0001)$ for both times. Scores for reliability of pain relief were better for the FPNS group, as were acceptability after the last treated episode and ease of use of the spray formulation in terms of convenience and satisfaction. Adverse effects were greater in the FPNS group. Systemic adverse effects consisted of vomiting $(10.6 \%)$, nausea $(8.8 \%)$, and dizziness $(8.0 \%)$. Local effects consisted of epistaxis (4.4\%) and nasopharyngitis (3.5\%). Two deaths in the treatment group and one in the placebo group were believed to be unrelated to the drug.

\section{Long-term safety of intranasal fentanyl}

Portenoy et $\mathrm{al}^{24}$ assessed the safety aspects of fentanyl pectin for cancer-related breakthrough pain. Patients in the study had chronic cancer pain requiring at least $60 \mathrm{mg}$ of oral morphine equivalent per day and had one to four attacks of breakthrough pain per day. Individual dose titration identified the appropriate breakthrough dose. Dose changes were performed as necessary to optimize comfort, but $90 \%$ did not require a dose change. After reviewing the adverse effects of 42,000 episodes of breakthrough pain in 110 patients, $24 \%$ experienced adverse effects that were systemic in nature and characterized as mild. There were no significant local nasal effects. 


\section{Intranasal versus oral transmucosal fentanyl citrate}

Mercadante et $\mathrm{al}^{25}$ conducted an open-label crossover trial comparing intranasal with oral transmucosal fentanyl citrate (OFTC). Patients with cancer pain were screened for breakthrough cancer pain and, if eligible, received a test dose of fentanyl $50 \mu \mathrm{g}$. Patients whose pain was opioid responsive were entered into the study and randomized to receive INFS or OFTC. In the study, individual titration was conducted to identify a dose that could successfully treat three of four episodes of breakthrough pain. There was then a final phase lasting approximately 2 weeks, during which the identified doses with each formulation were used and compared with each other for onset of meaningful pain relief, pain intensity, and use. With regard to the intranasal fentanyl, patients could receive a second dose of intranasal analgesic if there was no pain relief after 10 minutes. If the second dose did not help, rescue analgesics were allowed 10 minutes after the second intranasal spray. More rescue analgesics were allowed at 45 and 60 minutes, depending on whether the second intranasal dose was required. The primary outcome was the (stopwatch) measured time to patient-reported meaningful pain relief for either the OFTC or intranasal route of administration. Pain intensity (NRS 0-10) was also measured at time points 5, 10, 15, 20, 30, and 60 minutes after administration of INFS or OTFC. If a rescue dose was taken at 60 minutes, the preceding pain intensity value was carried forward. PID was measured at 5-minute intervals for the first 30 minutes, and the sum of PID was calculated for the 0-15 minute and 0-60 minute intervals.

Categorical scales were used to describe ease of use. Treatment preferences were recorded after the patients completed both arms. The investigators calculated the relationship between doses required for breakthrough pain and scheduled analgesic doses. Finally, the proportion of patients with a greater than $33 \%$ and a greater than $50 \%$ reduction in pain intensity score was also calculated for each treatment at 5, 10, 15, 20, 30, and 60 minutes postdosing. One-hundred and thirty-nine patients were enrolled in the trial, but 53 did not complete the titration phase. Rates of withdrawal were the same between groups. A total of 577 breakthrough pain episodes were treated with INFS, and 577 episodes were treated with OFTC. Ninety-three percent of patients treated six breakthrough episodes with INFS, and $92 \%$ were treated with OFTC. Nearly equal numbers of patients were able to find effective doses of INFS and OFTC (85\% and $87 \%$, respectively). In the INFS group,
$75 \%$ needed doses of $100-200 \mu \mathrm{g}$, and $60 \%$ in the OFTC group needed 200-400 $\mu \mathrm{g}$ doses for adequate breakthrough coverage. The investigators found a weak association between the amount of INFS taken and the amount of OFTC used for breakthrough pain, in that patients requiring higher INFS doses also needed higher OFTC doses, and those requiring lower doses of INFS needed lower doses of OFTC. Results in respect of the primary endpoint showed that the median time to meaningful pain relief was 11 minutes for INFS and 16 minutes for OFTC. More patients in the INFS group experienced a faster onset of pain relief than the OFTC group $(P<0.001)$. PID at 10 and 30 minutes was greater for the INFS group than for the OFTC group $(P<0.001)$ and at other times, such as $5,15,20$, and 60 minutes postdosing. It appeared that the PIDs were in favor of the INFS group for 60 minutes postdosing $(P<0.001)$. Statistically significantly greater proportions of the INFS group achieved 33\% and $50 \%$ reductions in pain scores than the OFTC group. There was a general impression of favorability that was greater with the INFS group. Fifty-six percent of patients experienced at least one adverse effect. The main adverse effect occurring in each arm of the study at a frequency of more than 5\% was nausea. Most adverse effects were considered mild. The severe adverse effects were few in number and consisted of a slightly greater number in the INFS group (severe nausea and vomiting). One patient developed nasal ulcers in the INFS group, and the INFS was discontinued with subsequent healing of the wounds. Patients preferred INFS over OFTC $(P<0.001)$ and also found INFS to be easier to use than OFTC.

\section{Postoperative pain in adults using INFS versus weak opioids}

Striebel et $\mathrm{al}^{26}$ evaluated the effects of intranasal fentanyl versus weak opioids as postoperative analgesic therapy in 20 patients receiving orthopedic procedures in a pilot study. On the first postoperative day, patients who had a postoperative pain score of 40 on a visual analog scale (VAS) were randomized to receive either intranasal fentanyl at $0.025 \mathrm{mg}$ every 6 minutes for 4 hours or "standard ward therapy" consisting of agents such as pethidine, tramadol, metamizole, codeine, and diclofenac as single agents or in combination, followed by the alternative regimen. Pain was measured every 30 minutes by VAS, after randomization, up to 480 minutes. The results in both arms of the study showed effective pain control at 30 minutes, but, as time elapsed, pain levels were lower in the intranasal fentanyl arm. Patients also expressed greater satisfaction with fentanyl. 


\section{Intravenous fentanyl versus intranasal fentanyl}

Patient-controlled analgesia (PCA) with intranasal fentanyl was compared with intravenous fentanyl in the postoperative period in 48 patients undergoing surgery for abdominal, orthopedic, or thyroid surgery. ${ }^{27}$ Eligible patients had to have a pain score of at least 40 on the VAS. Randomization was to fentanyl $25 \mu \mathrm{g}$ intranasally and fentanyl $17.5 \mu \mathrm{g}$ intravenously, with lock-out intervals of 6 minutes. Blinding was achieved by double-dummy design, whereby each patient had an intravenous PCA device and an intranasal PCA device. When in pain, the patient activated both routes and received either fentanyl or saline. Pain intensity was measured by NRS for the duration of the 240-minute study. In the intranasal group, the first reduction in pain score was $21 \pm 11$ minutes (range 15-45 minutes), and in the intravenous group was $22 \pm 16$ minutes (range 15-90 minutes). Overall, there were no differences in overall pain intensity, and patient-rated intranasal analgesia was rated as "excellent" or "good". Intranasal fentanyl was also compared with intravenous fentanyl for the management of acute and breakthrough pain in patients undergoing molar extractions. ${ }^{28}$ Patients were randomized to one of four doses $(75,100,150$, or $200 \mu \mathrm{g})$ of fentanyl by both intranasal and intravenous routes randomly after two separate extractions at 1 or more weeks apart, with pain scores on an NRS measured before administration and at $15,30,45,60,75,90,105,120,150,180,210$, and 240 minutes. Patients indicated time to meaningful pain relief and duration of analgesia. Plasma fentanyl levels were assessed before and at 1, 3, 5, 7, 9, 12, 15, 25, 40, 60, 90, 120, and 180 minutes after administration. Twenty-four patients were enrolled. $\mathrm{T}_{\max }$ was delayed compared with intravenous dosing (12.8 and 6.0 minutes $[P<0.001]$ ), and onset of analgesia was slower for intranasal analgesia (7 and 2 minutes $[P<0.001])$, but duration of analgesia did not differ between groups. There was a relationship between duration of effect and intranasal dose, and there was less use of breakthrough analgesia after the intranasal fentanyl $(P<0.005)$. Both groups tolerated the therapies well.

\section{Pediatric analgesia}

Intranasal fentanyl was compared with intravenous fentanyl in pediatric patients after abdominal surgery. ${ }^{29}$ Postoperatively, 36 patients with pain scores greater than 6 according to an objective pain scale (OPS) were randomized into one of two groups. Group A $(\mathrm{n}=17)$ received $0.5 \mathrm{mg} / \mathrm{kg}$ of intranasal fentanyl, followed by an intravenous injection of sodium chloride ( $1 \mathrm{~mL}$ of $0.9 \%)$. Patients in group $\mathrm{B}(\mathrm{n}=19)$ received intranasal normal saline ( $1 \mathrm{~mL}$ of $0.9 \%$ ), followed by intravenous fentanyl $0.5 \mu \mathrm{g} / \mathrm{kg}$. Pain scoring was conducted 5 minutes later, and those with scores of 2 or higher received the same protocol once, with scoring conducted every 1 minute. This was repeated 5 minutes later if scores were 2 or higher once again. The goal of such titration was to get to an OPS score of 2 or lower. Sixty minutes after the first administration, the primary scheme was carried out once per hour until 24 hours later. There were no differences between groups in time to achieve a score of less than 2 . There was a greater amount of fentanyl used in group A. There were more adverse effects consisting of dizziness (32\%) and nausea $(21 \%)$ in group B than in group A (12\% dizziness and $32 \%$ nausea $[P<0.05])$. Pain scores were equally reduced in both groups at 4 hours postadministration.

\section{Other surgery and pediatrics}

INFS has been used for analgesia in pediatric patients undergoing myringotomy. ${ }^{30}$ When given in conjunction with anesthesia, there is less postoperative agitation ${ }^{31}$ and analgesia that is equivalent in potency to other modalities such as nerve block. ${ }^{32}$

\section{Intranasal fentanyl versus morphine}

Several studies have been carried out comparing intranasal fentanyl with morphine. An emergency room-based, prospective, randomized, blinded, placebo-controlled study ${ }^{33}$ was carried out to evaluate the efficacy of intravenous morphine, intranasal placebo, and intranasal fentanyl for long bone fractures. Sixty-seven children were randomized to intravenous morphine $(10 \mathrm{mg} / \mathrm{mL})$ and intranasal placebo or intranasal fentanyl $(150 \mu \mathrm{g} / \mathrm{mL})$ and intravenous placebo. Pain scores by VAS were measured at $0,5,10,20$, and 30 minutes with adverse effects recorded. Thirty-four children received intravenous morphine and 33 received intranasal fentanyl. There were no differences in pain intensity reduction between the groups $(P=0.333)$ at 5 minutes and at time intervals thereafter. The mean dose of INFS was $1.7 \mu \mathrm{g} / \mathrm{kg}$ and the mean morphine dose was $0.11 \mathrm{mg} / \mathrm{kg}$. Another comparison with morphine was conducted in burn patients where 26 adult patients (22 males) with burn surface areas ranging from $1 \%$ to $25 \%$ were randomized to a placebo-controlled, crossover study over two periods and two treatments. ${ }^{34}$ Patients requiring identical wound management on consecutive days were randomized to patient-controlled intranasal fentanyl with oral placebo or oral morphine with intranasal placebo on the first day, with the alternating sequence on the subsequent day. 
Patients reported their pain intensity on an NRS before, during, and after the procedure. The mean dose of intranasal fentanyl given was $1.48 \pm 0.57 \mu \mathrm{g} / \mathrm{kg}$ and $0.35 \pm 0.12 \mathrm{mg} / \mathrm{kg}$ of oral morphine. There were no differences in pain scores between the groups. Two patients in the oral morphine group experienced hypotension, but there was no respiratory depression. Three patients required rescue analgesia, two cases involving morphine and one involving fentanyl.

\section{Other uses Hospice}

The hospice setting may be a place where intranasal routes of administration can have benefit. Twelve hospice patients ${ }^{35}$ were evaluated for the analgesic effects of $20 \mu \mathrm{g}$ of fentanyl for cancer-related breakthrough pain. Pain was rated by VAS before and 3, 5, 10, 15, 30, 45, and 60 minutes after receiving the dose. Eight of 12 experienced a reduction in pain score, four achieved an analgesic response after 5 minutes, and seven achieved an analgesic response within 10 minutes of receiving the dose. Nine patients felt that the analgesia obtained with the intranasal formulation was "good" or "very good". Two patients experienced local nasal itching or discomfort on first use, but this disappeared with time. Of the three patients who did not experience a good response with the intranasal fentanyl, one had a fracture and two had what was described as "relatively higher baseline opioid doses". A series of cases has shown efficacy for the use of intranasal fentanyl for the management of dyspnea in hospice patients. ${ }^{36}$ The cases were notable because the intranasal fentanyl was administered by nasal spray and had a rapid onset on action, usually within 1 minute, with good relief obtained in 1-4 minutes. There was no worsening of pulmonary function during administration of the intranasal fentanyl with any of the cases. Individual titration of the appropriate dose for dyspnea is necessary.

\section{Methods of administration}

Fentanyl doses have been administered in one or both nostrils using fentanyl solutions of $0.5,1.0$, and $2.0 \mathrm{mg} / \mathrm{mL}$, corresponding to doses of 50,100, and $200 \mu \mathrm{g}$ of fentanyl. ${ }^{15}$ The clinical trials for breakthrough pain suggest the use of nasal spray devices, which can provide more uniform dosing with patients.

\section{Conclusion}

The intranasal route of opioid administration shows great promise as an alternative to the traditional routes of administration. It appears to be especially compatible with highly lipophilic agents such as fentanyl. Overall, the use of the intranasal route appears to be of low burden to patients, is amenable for pain management for a variety of analgesic issues ranging from postoperative pain to cancer-related breakthrough pain, and is applicable to both adult and pediatric patient populations. In all likelihood, the intranasal route of opioid administration will replace the oral route as an option for several types of breakthrough pain.

\section{Disclosure}

The authors report no conflicts of interest in this work.

\section{References}

1. Patt RB, Burton AW. Pain associated with advanced malignancy, including adjuvant analgesic drugs in cancer pain management. Evaluation and Treatment of Chronic Pain. New York: Lippincott, Williams \& Wilkins; 1998:337-376.

2. Kalso E, Edwards JE, Moore RA, McQuay HJ. Opioids in chronic non-cancer pain: systematic review of efficacy and safety. Pain. 2004; 112:372-380

3. Dosen-Micovic L, Ivanovic M, Micovic V. Steric interactions and the activity of fentanyl analogs at the [mu]-opioid receptor. Bioorg Med Chem. 2006;14:2887-2895.

4. Shelley K, Paech MJ. The clinical applications of intranasal opioids. Curr Drug Deliv. 2008;5:55.

5. Vyas TK, Shahiwala A, Marathe S, Misra A. Intranasal drug delivery for brain targeting. Curr Drug Deliv. 2005;2:165-175.

6. Dale O, Hjortkjaer R, Kharasch ED. Nasal administration of opioids for pain management in adults. Acta Anaesthesiol Scand. 2002; 46:759-770.

7. Birkhoff M, Leitz M, Marx D. Advantages of intranasal vaccination and considerations on device selection. Indian J Pharm Sci. 2009;71:729.

8. Veldhorst-Janssen NML, Fiddelers AAA, van der Kuy PHM, et al A review of the clinical pharmacokinetics of opioids, benzodiazepines, and antimigraine drugs delivered intranasally. Clin Ther. 2009; 31:2954-2987.

9. Schwagmeier R, Oelmann T, Dannappel T, Striebel HW Patientenakzeptanz gegennber der patientenkontrollierten intranasalen Analgesie (PCINA). Der Anaesthesist. 1996;45:231-234.

10. Pennington AK, Ratcliffe JH, Wilson CG, Hardy JG. The influence of solution viscosity on nasal spray deposition and clearance. Int J Pharm. $1988 ; 43: 221-224$

11. Illum $L$, Jorgensen $H$, Bisgaard $H$, et al. Bioadhesive microspheres as a potential nasal drug delivery system. Int J Pharm. 1987;39:189-199.

12. Veldhorst-Janssen NML, Fiddelers AAA, van der Kuy PHM, et al. Pharmacokinetics, analgesic effect, and tolerability of a single preprocedural dose of intranasal fentanyl in patients undergoing drain removal after breast reduction or augmentation surgery: a prospective, randomized, double-blind, placebo-controlled study. Clin Ther. 2010; 32:1427-1436.

13. Paech MJ, Lim CB, Banks SL, et al. A new formulation of nasal fentanyl spray for postoperative analgesia: a pilot study. Anaesthesia. 2003;58:740-744

14. Grape S, Schug SA, Lauer S, Schug BS. Formulations of fentanyl for the management of pain. Drugs. 2010;70:57-72.

15. Panagiotou I, Mystakidou K. Intranasal fentanyl: from pharmacokinetics and bioavailability to current treatment applications. Expert Rev Anticancer Ther. 2010;10:1009-1021.

16. Christrup LL, Foster D, Popper LD, et al. Pharmacokinetics, efficacy, and tolerability of fentanyl following intranasal versus intravenous administration in adults undergoing third-molar extraction: a randomized, double-blind, double-dummy, two-way, crossover study. Clin Ther. 2008;30:469-481. 
17. Fisher A, Watling M, Smith A, Knight A. Pharmacokinetic comparisons of three nasal fentanyl formulations; pectin, chitosan and chitosanpoloxamer 188. Int J Clin Pharmacol Ther. 2010;48:138.

18. Prommer E. The role of fentanyl in cancer-related pain. J Palliat Med. 2009; 12:947-954.

19. Portenoy RK, Hagen NA. Breakthrough pain: definition, prevalence and characteristics. Pain. 1990;41:273-281.

20. Coluzzi PH, Schwartzberg L. Breakthrough cancer pain: a randomized trial comparing oral transmucosal fentanyl citrate (OTFC) and morphine sulfate immediate release (MSIR). Pain. 2001;91:123-130.

21. Portenoy RK, Taylor D, Messina J, Tremmel L. A randomized, placebo-controlled study of fentanyl buccal tablet for breakthrough pain in opioid-treated patients with cancer. Clin J Pain. 2006; 22:805.

22. Kress HG, Oronska A, Kaczmarek Z, et al. Efficacy and tolerability of intranasal fentanyl spray 50 to 200 [mu] g for breakthrough pain in patients with cancer: a phase III, multinational, randomized, doubleblind, placebo-controlled, crossover trial with a 10-month, open-label extension treatment period. Clin Ther. 2009;31:1177-1191.

23. Taylor D, Galan V, Weinstein SM, et al. Fentanyl pectin nasal spray in breakthrough cancer pain. J Support Oncol. 2010;8:184.

24. Portenoy RK, Raffaeli W, Torres LM, et al. Long-term safety, tolerability, and consistency of effect of fentanyl pectin nasal spray for breakthrough cancer pain in opioid-tolerant patients. J Opioid Manag. 2010;6:319.

25. Mercadante S, Radbruch L, Davies A, et al. A comparison of intranasal fentanyl spray with oral transmucosal fentanyl citrate for the treatment of breakthrough cancer pain: an open-label, randomised, crossover trial. Curr Med Res Opin. 2009;25:2805-2815.

26. Striebel HW, Olmann T, Spies C, Brummer G. Patient-controlled intranasal analgesia (PCINA) for the management of postoperative pain: a pilot study. J Clin Anesth. 1996;8:4-8.

27. Toussaint S, Maidl J, Schwagmeier R, Striebel HW. Patient-controlled intranasal analgesia: effective alternative to intravenous PCA for postoperative pain relief. Can J Anaesth. 2000;47:299-302.
28. Christrup LL, Foster D, Popper LD, et al. Pharmacokinetics, efficacy, and tolerability of fentanyl following intranasal versus intravenous administration in adults undergoing third-molar extraction: a randomized, double-blind, double-dummy, two-way, crossover study. Clin Ther. 2008;30:469-481.

29. Cheng X, Li JH. Effects of fentanyl administrated nasally and intravenously in post-operative analgesia in pediatric children: a comparative study of 36 cases. Zhonghua Yi Xue Za Zhi. 2008;88:2898.

30. Galinkin JL, Fazi LM, Cuy RM, et al. Use of intranasal fentanyl in children undergoing myringotomy and tube placement during halothane and sevoflurane anesthesia. Anesthesiology. 2000;93:1378.

31. Finkel JC, Cohen IT, Hannallah RS, et al. The effect of intranasal fentanyl on the emergence characteristics after sevoflurane anesthesia in children undergoing surgery for bilateral myringotomy tube placement. Anaesth Analg. 2001;92:1164.

32. Voronov P, Tobin MJ, Billings K, et al. Postoperative pain relief in infants undergoing myringotomy and tube placement: comparison of a novel regional anesthetic block to intranasal fentanylûa pilot analysis. Pediatric Anesthesia. 2008;18:1196-1201.

33. Borland M, Jacobs I, King B, O'Brien D. A randomized controlled trial comparing intranasal fentanyl to intravenous morphine for managing acute pain in children in the emergency department. Ann Emerg Med. 2007;49:335-340.

34. Borland ML, Bergesio R, Pascoe EM, et al. Intranasal fentanyl is an equivalent analgesic to oral morphine in paediatric burns patients for dressing changes: a randomised double blind crossover study. Burns. 2005;31:831-837.

35. Zeppetella G, O’Doherty CA, Collins S. Prevalence and characteristics of breakthrough pain in cancer patients admitted to a hospice. J Pain Symptom Manage. 2000;20:87-92.

36. Sitte T, Bausewein C. Intranasal fentanyl for episodic breathlessness. J Pain Symptom Manage. 2008;36:e3.
Patient Preference and Adherence

\section{Publish your work in this journal}

Patient Preference and Adherence is an international, peer-reviewed, open access journal focusing on the growing importance of patient preference and adherence throughout the therapeutic continuum. Patient satisfaction, acceptability, quality of life, compliance, persistence and their role in developing new therapeutic modalities and compounds to

\section{Dovepress}

optimize clinical outcomes for existing disease states are major areas of interest. This journal has been accepted for indexing on PubMed Central. The manuscript management system is completely online and includes a very quick and fair peer-review system. Visit http://www.dovepress.com/ testimonials.php to read real quotes from published authors. 